\title{
Metaphor and the development of pragmatic competence
}

\begin{abstract}
Language development among pre-school children will take place in all aspects of the language especially pragmatics, for instance, the ability to tell stories and semantics, understand the meaning of the language widely including the abstract forms. Their vocabulary will be widened which enable them to construct long and complex sentences and building up words using correct affixes. Language development at pre-school age is more creative, whereby the language creativity is enhanced by the surroundings, experiences, the metaphor that was developed through film and many other language activities. They use their developed language creativity when they communicate with friends and people in their society. Among the pragmatic competence that they have developed are pronoun, similes and metaphor language. Therefore, in this study, focus will be on the language usage in children's speech. The aspect of the language usage will converge on the use of symbol language used by the children to convey message, depict mind and emotion. In this study, the samples of the study are 25 Malay pre-school children. The method of the study is observation. The language data were recorded during their morning dialogue session, story telling, games and their morning tour around the kindergarten. The collected data was analyzed using the pragmatic theory, the theory of Relevance.
\end{abstract}

Keyword: Metaphor, Pragmatic Competence 